\title{
Corporate Social Network Recruitment in the Age of Big Data
}

\author{
Wei Wei \\ Guizhou University of Finance and Economics Business \\ School \\ Guiyang City, Guizhou Province
}

\author{
Wang JianJu \\ Guizhou University of Finance and Economics Business \\ School \\ Guiyang City, Guizhou Province
}

\begin{abstract}
Nowadays, social networks has become a part of our lives. The value of early social networks was limited to the social world, and with the popularity of modern Internet thinking, social networks were given a considerable weight of business functions, more and more companies began to perform recruitment by social networks. In the process of mass recruitment information, big data technology has become an indispensable technical tool. This paper compares the new social network recruitment model in the big data era with the traditional online recruitment model, summarizes the advantages of social networking recruitment and the problems thereof. It further studies the specific application of big data in social network recruitment, and puts forward the challenges and suggestions of social network recruitment in the era of big data.
\end{abstract}

Keywords-Social network; Online recruitment; Big data analysis process; Traditional online recruitment

\section{INTRODUCTION}

In the Internet era, technology is the foundation of creating value. Talents have become the magic weapon of competition among modern enterprises. Recruitment is the basic way for enterprises to absorb talents. Therefore, it is of vital importance to find a fast and efficient way of recruitment for the survival and long-term development of enterprises.

In recent years, "big data" has become a popular word in the Internet era. Big data represents not only an advanced Internet thinking, but also an indispensable technology in the Internet era. Faced with the complicated process of recruitment, social network recruitment will inevitably produce a large number of complex data, so it will be big. The application of data technology in network recruitment to form a new recruitment model is a trend of future development. For enterprises, we should follow the trend and make bold innovations and improvements in talent introduction to create opportunities for their own long-term development.

\section{COMPARATIVE ADVANTAGE ANALYSIS ON SOCIAL NETWORK RECRUITMENT AND TRADITIONAL ONLINE RECRUITMENT}

\section{A. Different scope of influence}

The influence of social network recruitment and traditional online recruitment actually depends on the attributes of their respective users.

From the perspective of job-seekers' desire, the traditional online recruitment website only has the function of recruiting job search, and only those who have strong desire to seek employment will $\log$ on to the website to find job opportunities. The social network is different from its own function is social networking, social networking sites have a number of users than professional recruitment sites can not match, the broader user coverage can not only enable companies to recruit strong initiative job seekers, The complex social relationships can also be- used to target potential passive candidates, helping companies to form a strong pool of talent.

From the level of job seekers, professional recruitment websites are only suitable for the recruitment of talents at the middle and low end of basic posts, because most of the users who use professional recruitment websites to apply for jobs are new graduates at a lower level. They enter the workplace for the first time and lack a network of people. Job-seeking channels are inevitably single, so they prefer such highly professional recruitment sites. For high-end talent or talented people, because of the confidentiality of their professional information, they do not choose such professional recruitment sites, usually by specialized headhunting companies for one-way contact. Enterprises can communicate with this kind of talents through social network, on the one hand, it can be used for enterprises. Save the cost of headhunting companies, but also improve the success rate of the enterprise to accept talent.

Under the background of global economic integration and the globalization of talent flow, the global recruitment activities are heating up, and the recruitment of international talents has put forward higher requirements for recruitment enterprises and recruitment websites. However, the current professional recruitment website on the market does not have the corresponding hardware and software conditions, can not meet the recruitment of international talent enterprises, and social networks can solve this problem through a complex network.

\section{B. Different modes of action}

In general, the traditional online recruitment operation includes enterprises, professional recruitment websites and job seekers. In general, the mode of operation is that the enterprises put the recruitment information on the recruitment website, and the candidates also submit their job search resumes to the website. The website creates its own resume database and carries out preliminary screening according to the requirements of the enterprise. Finally, the company will interview candidates who meet the requirements. Therefore, in this mode, the professional recruitment website mainly acts as an 
intermediary, play a "tool" role [10], in the initial recruitment of enterprises and candidates are independent of each other, only to the later interview links, enterprises and should It is only between the candidates that real contact begins.

In addition to companies, social networking sites and job seekers, social network recruiters include referrals that provide networking. Internal referrals refer to the employees of an enterprise, while external referrals mainly refer to corporate outsiders who have a large network of people in social networks. The operating mode of social network recruitment is different from traditional online recruitment in that companies will provide a certain amount of bonus for referrals, who will recommend the right people from their own network to the social network. In this process, the influence of networking circles is far from the traditional recruitment website; In addition, communication between companies and candidates does not have to wait until the interview At the same time, enterprises can log on to the candidate's home page and judge the authenticity of their personal characteristics and resume by observing the online behavior of the candidates. In the process, social networking sites play a "platform" role, not only providing companies with multiple opportunities to learn about candidates, but also drawing potential job seekers into hiring relationships through referrals. Improve the authenticity and success rate of recruitment.

\section{Differences in the effectiveness of recruitment}

Recruitment effectiveness includes the efficiency and effectiveness of recruitment. Efficiency refers to the length of time span and the cost of recruitment, and the effect refers to the success rate of recruitment and the quality of recruiting talent.

In terms of efficiency, the time span of social network recruitment is shorter than that of traditional online recruitment. Traditional online recruitment companies simply post recruitment information to recruitment websites, and then passively wait for candidates to apply. Although it may seem labor-saving, the time span of recruitment depends entirely on the speed at which candidates receive information and how quickly the site matches it; For social network recruitment, the recruitment information of enterprises can be transmitted quickly by forwarding, and the enterprises also change from passive waiting to active attacks, and the two-way circulation of search information greatly shortens the time of recruitment.

The cost of social network recruitment is lower than that of traditional online recruitment. Professional recruitment websites make money by charging registered companies membership or traffic fees, which can be costly to gain a favorable advertising position on the site. By contrast, social networking sites are free for companies to open, companies can publish recruitment information at zero cost, even if there is a fee is also a result of the charging model, more likely to be favored by enterprises.

In terms of effect, social network recruitment is realized on the basis of networking, and has the characteristics of precise positioning, that is, references are always targeted to search for talent according to the requirements of the enterprise. Therefore, the recommended personnel have initially met the standards of the enterprise. The possibility of hiring the candidate is also increasing. Second, companies can use social networking sites to track candidates' background deeply, which helps enterprises to have a deeper understanding of candidates and better guarantee the quality of recruiting talent compared with traditional online recruitment.

\section{RESEARCH ON THE CURRENT SITUATION AND PROBLEMS OF SOCIAL NETWORK RECRUITMENT}

\section{A. The status of Social Network recruitment}

In a 2014 iResearch survey of social media employer brand promotion and corporate recruitment applications, the survey found that 8 percent of companies had no plans to use social media for the time being, according to the survey on how many companies were using social media. The planned rate is 14 percent, and the proportion of people using social media is 78 percent, using and planning to use social media to reach 92 percent. Compared with previous years, the rate was 25 per cent in 2011,52 per cent in 2012 and 84 per cent in 2013, although it was the highest in recent years. New data are not available, but the growth in this proportion is enough to see how much mobile Internet is being used across the business market.

According to the 40th "Statistics report on the Development of China's Internet Network" released by the China Internet Information Center (CNNIC) in Beijing in August 2017, the number of Internet users in China reached 751 million as of June 2017. Of these, $80 \%$ of Chinese netizens regularly visit social networks, or 662 million, and in a social network based on this, you may be only one person apart from any stranger or the information you need. In the face of such a large number of users, such a complex network of contacts, it is enough to see the future development of social network recruitment. It will be immeasurable.

At present, there are many different levels of recruitment websites in the market, the high-end ones are neighboring, skyline, pulse; the middle end of the recruitment are 51 job, main Street, Zhaopin; the low end of the market, 58.com and so on. They represent traditional online recruitment, and social network recruitment, because of its more obvious nature on the Internet, depends on interpersonal relationships rather than the characteristics of professional recruitment and is independent of professional recruitment sites. At present, the main channel in the domestic market is through WeChat, Weibo and Renren.

\section{B. Research on Social Network problems}

\section{1) Limitation of talent level}

Because of the distinctive technical characteristics and technical characteristics of social network recruitment, the level of talents recruited is limited. At present, the people who use social recruitment mainly focus on young people and middle and higher talents, and for those who are not good at using the Internet, The advantage of social recruitment has been greatly weakened. Therefore, using social network alone can not complete the recruitment task of enterprises, must be combined with traditional recruitment, such as some low-skilled workers, production workers must adopt the traditional recruitment model. 


\section{2) Immature Big data technology}

As we all know, WeChat and Weibo have very low barriers to entry. They can enter the ecosystem only by using their smartphones. Any individual or institution can register. There are active people in the WeChat circle, regardless of their academic qualifications. There is no distinction between good and bad. In the face of a large number of users, if the technology of the enterprise is not mature, it is difficult for the enterprise to identify the users with real needs. In this case, all the advantages of social network recruitment will become one of the most difficult problems in the enterprise.

\section{RESEARCH ON THE APPLICATION OF BIG DATA TECHNOLOGY IN SOCIAL NETWORK RECRUITMENT}

The basic idea of big data's application in the social network is nothing more than the social network recruitment process and then supplemented by the data processing flow of big data technology, so that big data technology can be applied to every link of recruitment scientifically. Big data's data processing process consists of data acquisition, data processing and integration, data analysis, data interpretation. The process of designing network recruitment for enterprises is to publish Recruitment Information, preliminary screening of application information, further screening and identifying candidates for online examination, identifying recruitment list, optimize the recruitment platform of the enterprise, so that the two processes cooperate with each other. So the organic combination of the two processes is: data acquisition corresponding to the enterprise to issue recruitment information, data processing integration corresponding to the initial screening of enterprise application information, data analysis of the corresponding enterprise search suitable talent, data interpretation corresponding to the enterprise optimization recruitment platform.

\section{A. Establishing a social network platform suitable for enterprises}

The social network platform is the infrastructure for social recruitment. The success of the platform will directly affect the final recruitment results. In the process of building a social network platform, enterprises should pay attention to the following points: first, enterprises should understand the user characteristics of different social platforms, including age characteristics, when they register their corporate homepage of WeChat, Sina Weibo and Renren. The level characteristic, the social characteristic and so on; Secondly, in order to give full play to the advantages of social network platform recruitment and expand the data sources of recruitment, enterprises should implement online recruitment channels and internal employee recommendation channels. Specific mode of operation It is: requiring employees to $\log$ on to the enterprise's online recruitment system, and to contact their social platform by forwarding their recruitment information or by directly recommending suitable personnel within their own interpersonal circle, and setting up a corresponding incentive mechanism. If the candidate is accepted, the entire recommendation chain of employees will achieve the corresponding rewards, specific incentives should be set according to the specific situation of the enterprise itself; Finally, the enterprise home page of the social network platform and the subsequent information update is a process to be maintained. The enterprise should establish a set of standardized network recruitment system, invest special funds for it, and formulate appropriate propaganda strategy in time.

\section{B. Data acquisition-release of recruitment information}

The data collection in this link contains two levels of data. On the one hand, we must collect the data of existing employees in the enterprise and establish a database of talents in the enterprise. The most important of these is to analyze the age structure and educational background structure of each post The length of service structure, know which employees will be promoted, which employees will leave the post, at the same time according to the strategic objectives of human resources management and the enterprise's current talent allocation, to develop a talent recruitment plan in line with the actual situation of the enterprise; On the other hand, to collect social platform user data, different social platform users' education, age and level are different, such as the general situation WeChat suitable for campus recruitment; Weibo Renren suitable for social recruitment; LinkedIn suitable for high-end talent recruitment. However, enterprises should collect data and analyze the user characteristics of different platforms, and publish recruitment information for the platform that is most suitable for different job talent selection and enterprise recruitment plan, which can further improve the satisfaction of the candidates.

\section{Data processing and Integration-preliminary screening of candidate Information}

The focus of this link is that enterprises need to set up basic parameters and matching parameters. The so-called basic parameters refer to the minimum threshold of posts, such as age, education, foreign language level, etc. The matching parameter is the selection of the matching degree between the employee and the post on the basis of satisfying the basic parameters. The selection criteria and parameter setting should be completed jointly by the personnel department and the human resources department. The employment department sets the parameter from the competence angle, the manpower department mainly sets the parameter from the applicant's comprehensive quality and the enterprise environment fusion angle. This link adopts the method of man-machine combination and the selection of cumulative bonus points between basic parameters and matching parameters. The standard completion of the candidate's primary election.

\section{Data analysis-better identification of suitable candidates}

After using the screening criteria to complete the preliminary screening of candidates, enterprises need to further select more suitable candidates to enter the offline interview process, in which the core technology of big data's technology, that is, talent radar technology, should be used. Talent radar technology is mainly based on the in-depth analysis of various social network data to construct the interest map, personality map, skill map, behavior map and relationship map of the candidates. From these maps, the author's personality characteristics, interests, hobbies and social circles are extracted, and "professional matching, personality matching and industry shadow" are given according to the evaluation criteria of the job competency of the enterprise. According to 
the different weights of each dimension, such as response force and trust relationship, the comprehensive score of the candidates is obtained, and the list of candidates entering the offline interview is determined according to the comprehensive scores. The talent radar technology completely replaces the resume screening work of the human resources department, uses the scientific technology to replace the artificial subjective choice, uses big data technology to carry on the directional analysis, the horizontal match, greatly saves the resume screening time. The quality of the candidates was improved.

\section{E. Data explaination-optimizing enterprise network recruitment platform}

In the recruitment model of social networks, determining the final recruitment list does not represent the end of the recruitment process, but also ensures that the final screening results are fully applied within the enterprise.

On the one hand, the recruitment results should be published on the company's internal network within the company, which contains key information such as the channels through which candidates can be obtained, the characteristics of candidates' competence, and the amount of rewards awarded to each reference person in the recommendation chain. The company also needs to update the talent structure of the enterprise talent database. A summary of the candidate's competency and the data obtained from the channel are crucial to the next recruitment of the company.

On the other hand, the enterprise network recruitment platform simultaneously undertakes the dual function of publishing recruitment information and setting up enterprise image, and maintaining the social network home page is also a work that can not be ignored. Big data technology can help enterprises analyze the length of stay of candidates in various pages of the recruitment website and the number of views of text, pictures, and videos in the website. Enterprises can focus on building up their interest in candidates according to the final data. A reasonable distribution of text, pictures and videos.

Finally, companies can work strategically with the social networking platform to establish a database of candidates who belong to the company based on a large number of user information gathered on the platform, and to maintain long-term contact with them by phone, WeChat, e-mail, etc. After that, the vacant positions can be replenished in time, and the efficiency of recruitment can be improved.

\section{Challenges AND SUgGestions OF SOCIAL NETWORK RECRUITMENT IN BIG DATA ERA}

\section{A. Data security issues}

Social networking sites contain important information and personal information about recruitment companies and candidates. The data security of the sites is vital to both recruitment companies and candidates, and reasonable maintenance of the recruitment website system is not attacked by illegal elements or hackers. There are the following suggestions: first, the social network recruitment process to implement dynamic monitoring, the system vulnerability timely repair, once the virus invasion to take timely measures;
Second, using data encryption technology to encrypt the information that may be leaked in the website. If the website is invaded, the loss can also be minimized. Third, the firewall can continuously upgrade the service system Fend off some viruses and dangerous sites.

\section{B. Problem of Data dictatorship}

As data analysis becomes increasingly important in hiring decisions, recruiters will inevitably suffer from data authoritarianism, relying too heavily on data to ignore perceptions. Enterprises should realize that data, although scientific and intuitive, can not completely replace the role of human beings. Enterprises should learn to use data rationally and have the correct big data idea: in the Internet age, a decision must not be supported by data. But it is impossible to rely on data support alone to make decisions. Use all available information to make heartfelt decisions to avoid being lost in the sea of data.

\section{Security of Job seekers' Privacy}

Once data users discover big data's value to recruitment, they may further obtain and use personal data from job seekers, which inevitably contains personal information that job seekers do not want to use. Recruitment agencies may even disclose or sell out personal information for profit, threatening the privacy of job seekers. Data users, as the biggest beneficiaries, should be legally aware and accountable for their actions, which may be the best way to protect the privacy of job seekers.

\section{CONCLUSION}

In the Internet era, social network recruitment has become a new trend in the field of recruitment. Compared with traditional online recruitment, it has obvious advantages, although it still faces a lot of challenges and shortcomings in the current practical application. But it contains huge value and broad development space without doubt. With the continuous development and wide application of big data technology, the data value of social network will be better excavated, and social network recruitment will be better perfected with the help of big data technology. Finally, it becomes a standard and efficient recruitment method, which promotes the transformation of the whole human resources management industry.

\section{REFERENCES}

[1] He Jie. Social networking recruitment-A new approach to corporate recruitment [J]. Management and Management, 2013 (04): 40-42.

[2] Xiao Qiushui. Online recruitment skills [J]. Corporate Management, 2012 (08): 89-90.

[3] Chen Lin, Yuan Qinghong, Zhu Weimin. The present situation and suggestion of socialized recruitment in Enterprises: a case study of Weibo and Social networking site recruitment [J]. Human Resources Development in China, 2013 (03): 23-27 32.

[4] Liu Yang. Study on the influence of big data Technology on the CVs Collection stage in the online recruitment process $[\mathrm{J}]$.Modern Marketing: Xueyuan Edition, 2012 (6): 104-104.

[5] Wu Lin. How to understand the Times of big data? [J] Red Flag draft, 2015 (11): 39-42.

[6] Cheng Ling. On online recruitment in big data era [J]. Enterprise Reform and Management, 2016 (02): 51. 